\title{
Noninvasive prenatal screening for fetal aneuploidy, 2016 update: a position statement of the American College of Medical Genetics and Genomics
}

\author{
Anthony R. Gregg, MD, MBA'1, Brian G. Skotko, MD, MPP², Judith L. Benkendorf, MS³, \\ Kristin G. Monaghan, $\mathrm{PhD}^{4}$, Komal Bajaj, $\mathrm{MD}^{5}$, Robert G. Best, $\mathrm{PhD}^{6}$, Susan Klugman, $\mathrm{MD}^{7}$ and \\ Michael S. Watson, MS, $\mathrm{PhD}^{3}$; on behalf of the ACMG Noninvasive Prenatal Screening Work Group
}

\begin{abstract}
Disclaimer: This statement is designed primarily as an educational resource for clinicians to help them provide quality medical services. Adherence to this statement is completely voluntary and does not necessarily assure a successful medical outcome. This statement should not be considered inclusive of all proper procedures and tests or exclusive of other procedures and tests that are reasonably directed toward obtaining the same results. In determining the propriety of any specific procedure or test, the clinician should apply his or her own professional judgment to the specific clinical circumstances presented by the individual patient or specimen. Clinicians are encouraged to document the reasons for the use of a particular procedure or test, whether or not it is in conformance with this statement. Clinicians also are advised to take notice of the date this statement was adopted and to consider other medical and scientific information that becomes available after that date. It also would be prudent to consider whether intellectual property interests may restrict the performance of certain tests and other procedures.
\end{abstract}

Noninvasive prenatal screening using cell-free DNA (NIPS) has been rapidly integrated into prenatal care since the initial American College of Medical Genetics and Genomics (ACMG) statement in 2013. New evidence strongly suggests that NIPS can replace conventional screening for Patau, Edwards, and Down syndromes across the maternal age spectrum, for a continuum of gestational age beginning at 9-10 weeks, and for patients who are not significantly obese. This statement sets forth a new framework for NIPS that is supported by information from validation and clinical utility studies. Pretest counseling for NIPS remains crucial; however, it needs to go beyond discussions of Patau, Edwards, and Down syndromes. The use of NIPS to include sex chromosome aneuploidy screening and screening for selected copy-number variants (CNVs) is becoming commonplace

American College of Medical Genetics and Genomics (ACMG) guidelines and statements have assisted patients seeking prenatal screening information and health-care providers responsible for providing accurate and up-to-date information to their patients. ${ }^{1-3}$ Until recently, noninvasive prenatal screening for aneuploidy relied on measurements of maternal serum analytes and/or ultrasonography. These have a false-positive rate of approximately $5 \%$ and detection rates of $50-95 \%$, depending on the specific screening strategy used. Advances in genomic technologies led to noninvasive prenatal screening that relies on the presence of cell-free DNA derived from the placenta but circulating in maternal blood, which is referred to here as noninvasive prenatal screening (NIPS). Massive parallel sequencing because there are no other screening options to identify these conditions. Providers should have a more thorough understanding of patient preferences and be able to educate about the current drawbacks of NIPS across the prenatal screening spectrum. Laboratories are encouraged to meet the needs of providers and their patients by delivering meaningful screening reports and to engage in education. With health-care-provider guidance, the patient should be able to make an educated decision about the current use of NIPS and the ramifications of a positive, negative, or no-call result.

Genet Med advance online publication 28 July 2016

Key Words: cell-free fetal DNA; noninvasive prenatal testing; prenatal genetic screening

of maternal and placental (also called fetal when speaking of the fraction of this DNA in maternal blood) fragments of DNA occurs simultaneously. Sequencing with quantification can be random, targeted, and followed by quantification or exploitation of single-nucleotide polymorphisms. ${ }^{4-8}$ Alternatively, sequencing can take place by measuring the release of hydrogen ions as nucleotides are added to a DNA template (i.e., semiconductor sequencing). ${ }^{9}$ Microarray technology can also be used to quantify DNA. ${ }^{10}$ Bioinformatics that enable these methodologies is complex and proprietary. Since the introduction of NIPS in 2011, health-care providers and patients have experienced marketing pressures, rapidly evolving professional practice guidelines, and confusion regarding the appropriate role of

${ }^{1}$ Department of Obstetrics and Gynecology, University of Florida, Gainesville, Florida, USA; ${ }^{2}$ Department of Pediatrics, Harvard Medical School and Division of Medical Genetics, Department of Pediatrics, Massachusetts General Hospital, Boston, Massachusetts, USA; ${ }^{3}$ American College of Medical Genetics and Genomics, Bethesda, Maryland, USA; ${ }^{4}$ GeneDx, Gaithersburg, Maryland, USA; ${ }^{5}$ New York City Health + Hospitals/Albert Einstein College of Medicine, Bronx, New York, USA; ${ }^{6}$ University of South Carolina School of Medicine, Greenville Health System, Greenville, South Carolina, USA; ${ }^{7}$ Montefiore Medical Center, Department of Obstetrics \& Gynecology and Women's Health, Albert Einstein College of Medicine, Bronx, New York, USA. Correspondence: Anthony R. Gregg (greggar@ufl.edu)

The Board of Directors of the American College of Medical Genetics and Genomics approved this statement on 23 May 2016. 
NIPS in prenatal practice. ${ }^{11-15}$ This position statement replaces the 2013 "ACMG Statement on Noninvasive Prenatal Screening for Fetal Aneuploidy."3

We emphasize that all genetic screening has residual risk (i.e., risk of having a genetic condition even after receiving a negative or "normal" result). This concept is independent of the screening modality, condition screened, or number of conditions screened. The concept of residual risk supports our use of the acronym NIPS, where the "S" represents screening. It is important to emphasize what NIPS does not provide to patients. NIPS is not used clinically to screen for single-gene disorders (e.g., variation in the genome caused by relatively small changes in nucleotide sequence). NIPS is not used to predict late pregnancy complications. NIPS does not screen for open neural tube defects; therefore, maternal serum $\alpha$-fetoprotein testing to screen for open neural tube defects should still be offered at $15-20$ weeks of gestation. NIPS does not replace routine fetal anatomic screening using ultrasound.

Screening tests move through a predictable stepwise progression from laboratory development to clinical use. The ACMG recognizes this course as (i) analytical validity, (ii) clinical validity, and (iii) clinical utility. The last of these is the most complex.

- Analytical validity refers to whether the screening test detects the target of the test in those with the target (analytical sensitivity) without detecting it in those without the target (analytical specificity). Regarding NIPS, analytical validity asks whether various concentrations of maternal and placental DNA can be used to determine the presence or absence of fetal aneuploidy (or other conditions). Analytical validity has been established for the variety of screening methods discussed in this article. $^{10,16-19}$

- Clinical validity refers to how well NIPS performs and focuses on detection rate (DR), the proportion of those who screen positive and will have the clinical condition (clinical sensitivity), and the proportion who will not (clinical specificity (SPEC)). These test metrics are independent of the prevalence of the condition being screened. Because NIPS addresses fairly uncommon conditions, validation studies are used to understand the DR and SPEC using banked or research samples. This allows overrepresentation of samples for the target condition of interest. Between 2011 and 2013, there were at least eight widely quoted validation studies spread across four laboratories offering NIPS clinically. ${ }^{4-8,20-22}$ Validation studies reached similar conclusions. NIPS had very high DR and SPEC, reaching nearly 99\% for Down syndrome caused by trisomy 21, translocations, and trisomy 21 mosaicism. The DR and SPEC were $80-100 \%$ for Edwards syndrome caused by trisomy 18 and trisomy 18 mosaicism, as well as for Patau syndrome caused by trisomy 13, translocations and trisomy 13 mosaicism. In this document, we refer to all three syndromes as "traditionally screened aneuploidies." Thus, in clinical validation studies, NIPS was shown to outperform conventional screening approaches. ${ }^{23-25}$

- Clinical utility refers to whether a screening test is reliable and useful to patients. Clinical utility studies inform patients, providers, and payers about decision making. These studies can provide objective test metrics such as positive predictive values (PPVs) and negative predictive values (NPVs). It is noteworthy that PPV and NPV can be determined for a population by modeling (using DR and SPEC as well as population prevalence) or by actual measure. Furthermore, one can establish PPV on a population basis (e.g., all women of a certain age) or individually (using information that is patient-specific). Cost efficacy in terms of dollars or cost utility measured by cost per case detected or quality-adjusted life-year is also used to describe clinical utility. ${ }^{26}$ Because cost efficacy and cost utility studies use a high degree of modeling and assumptions (clinical care and monetary), these are at risk for bias (systematic and random). We chose not to include studies of this nature when making our recommendations.

\section{IMPLEMENTATION OF NIPS INTO PRACTICE: GENETIC TESTING AS A MULTIFACETED CLINICAL PROCESS}

Genetic testing and screening modalities used in pregnancy, such as NIPS, are offered with the aim of providing patients information that can help them optimize their pregnancy outcomes. ${ }^{27}$ It is accepted practice, when implementing these modalities, to follow a multifaceted process in which genetic counseling is a common thread. Specific steps include: pretest education, counseling, and informed consent; the screening or testing procedure; a laboratory component that includes test interpretation; and, finally, the disclosure of results to the patient within a context that includes the appropriate education, counseling, and follow-up.

The core of genetic counseling is establishing patient desire and expectations. Genetic counseling is not merely educational; it is a patient-centered form of medical communication facilitating decisions on a course of action that are made solely by the patient once the patient has been given the necessary facts, alternatives, and anticipated consequences. ${ }^{28,29}$ In this context, genetic counseling follows the Rogerian method, which is client-centered and nondirective. ${ }^{30} \mathrm{ACMG}$ recognizes it is beyond the scope of prenatal care providers to describe all genetic conditions amenable to diagnosis or screening in a pretest counseling session. However, an effort should be made to discuss in a general way the types of conditions that can (e.g., aneuploidy, translocations, microdeletions, and microduplications) and cannot (e.g., many single-gene disorders), be identified, including test limits in the case of the former, when a family history is unremarkable. $^{31}$ 
Patient preferences for information should play a pivotal role in guiding the use of NIPS in prenatal care. This is in keeping with generally accepted genetic counseling tenets and respects that clinical utility may vary between patients. ${ }^{28,29}$ Clinical utility includes test metrics (PPV and NPV), cost, and a patient's unique value system construct framed by (among other things) cultural traditions and religious beliefs. We recognize that this construct is not homogeneous across the United States. The desire for diagnostic testing or screening, the uptake of diagnostic testing, and decisions made when positive results are confirmed are influenced by a patient's value system. However, establishing a patient's value system construct can be complex and confusing. In the context of an evolving technology such as NIPS, the patient's ability to accept uncertainty with regard to possible screening outcomes should also be considered and explored as part of the pretest communication process. Cost plays a role in society's willingness to pay. Insurance coverage (private or public), responsibility for co-payments, and out-of-pocket expenses factor into the nature of follow-up diagnostic tests, availability of genetic counseling services, and reproductive decision making.

For the genetic testing and screening modalities used in pregnancy to provide patients with information that can help them optimize their pregnancy outcomes, patients must be allowed to make informed choices that occur across a time continuum. Prenatal screening and diagnostic testing target 20 weeks of gestation as an upper limit for implementation. ${ }^{32}$ Decision making is circumscribed by state-specific laws (e.g., 20 weeks), ${ }^{33}$ which highlights the importance of timely delivery and processing of accurate and complete information at each step. NIPS can be performed at an earlier gestational age than conventional screening, and there is no gestational age upper limit after 10 weeks of gestation. This means that patients can get the most accurate screening information at an earlier gestational age, thus enhancing informed decision making.

- ACMG recommends:

- Providing up-to-date, balanced, and accurate information early in gestation to optimize patient decision making, independent of the screening approach used.

- Laboratories work with public health officials, policymakers, and private payers to make NIPS, including the pre- and posttest education and counseling, accessible to all pregnant women.

For some patients the goal in prenatal screening may be to maximize the detection of fetal genetic diagnoses. In this scenario, fetal diagnostic testing (e.g., chorionic villous sampling or amniocentesis) followed by chromosomal microarray (CMA) using fetal DNA should be offered, and NIPS may not be the best choice. With diagnostic testing, whole-chromosome abnormalities, unbalanced chromosome rearrangements, small losses or gains of chromosomal material (CNVs), and in some cases single-gene disorders can be detected. An NIH study of prenatal CMA suggested the background rate of small clinically significant CNVs is $1-2 \%{ }^{34}$ Fetal diagnostic testing carries a small risk. Pregnancy loss rates before 24 weeks of gestation for amniocentesis range from 0.1 to $0.9 \%(1 / 1,000-1 / 111)$ and for chorionic villous sampling range from 0.2 to $1.3 \%$ (1/5001/77). ${ }^{35,36}$ Results from these studies reflect diagnostic testing performed because of abnormal ultrasound findings, positive aneuploidy screening, or other at-risk conditions. Therefore, one can conclude that these procedure-related miscarriages are overestimates of risk compared to selecting a procedure solely for obtaining maximal information.

Patients may prefer a screening test, and there are many to choose from. Conventional screening approaches such as firsttrimester screening, second-trimester screening, or combinations of both (e.g., stepwise sequential screening) have good detection rates (80-95\%) but high false-positive rates (3-5\%). Stepwise sequential screening has both $(\sim 95 \%$ and $\sim 5 \%)$ but is not universally used due to the required logistics. When choosing a conventional screening approach, patients should be aware of the high false-positive rate, which may lead to diagnostic procedures and, consequently, diagnoses not detected by NIPS (e.g., some chromosome abnormalities and CNVs). For patients who prefer to avoid diagnostic testing but desire highly accurate screening for Patau, Edwards, and Down syndromes, NIPS may be preferred. There are pros and cons to any screening approach. After careful counseling, patients will ideally select the paradigm that is most aligned with their goals. Prenatal care providers should try to understand the clinical utility construct of individual patients during the informed consent and decision-making processes.

- ACMG recommends:

- Allowing patients to select diagnostic or screening approaches for the detection of fetal aneuploidy and/or genomic changes that are consistent with their personal goals and preferences.

- Informing all pregnant women that diagnostic testing (CVS or amniocentesis) is an option for the detection of chromosome abnormalities and clinically significant CNVs.

\section{SHOULD NIPS BE OFFERED TO ALL PATIENTS, INCLUDING THOSE AT LOW OR AVERAGE RISK?}

In 2013, the ACMG was careful not to restrict NIPS to specific patient groups. ${ }^{3}$ Recent clinical utility trials ${ }^{23-25,37}$ compared NIPS to conventional screening methods for women at low risk or average risk compared to women at high risk. The DR, SPEC, PPV, and NPV for Patau, Edwards, and Down syndromes were reported. Clinical utility, measured as PPV and NPV in these studies, supports the earlier ACMG position, and several professional organizations have subsequently altered their positions. ${ }^{38-40}$ Data from two large studies show that for "low risk women", the PPV for Down syndrome after NIPS was $50-81 \%(\mathrm{~N}=55,244)^{24,25}$, and for "high risk women" this was $94 \%(\mathrm{~N}=72,382) .{ }^{25}$ NIPS and conventional screening were compared and showed NIPS was superior with regards to PPV (80.9 vs. $3.4 \%, \mathrm{~N}=15,841){ }^{24}$ The NPV approached $100 \%$ for Down 
syndrome in these studies. Similarly, for Patau and Edwards syndromes, the PPVs after NIPS (Patau 33-90\%, Edwards $50-70 \%)^{24,25}$ were superior to those with conventional screening (Patau 14\%, Edwards 3.4\%) ${ }^{24}$ and the NPV was $100 \%$ for both conditions. ${ }^{23,24}$

High PPV provides benefits to patients by enabling them to more easily weigh the advantages and disadvantages of followup diagnostic testing. Additional benefits of NIPS include earlier implementation with no gap across the gestational age spectrum, unlike conventional screening methods. This allows confirmatory diagnostic testing earlier in gestation and provides a screening option for patients who present for care any time after the first trimester. Earlier diagnosis facilitates providing up-to-date, balanced, and accurate information at a time that may enable patients to consider the broadest range of reproductive options. In some cases, patients will elect to alter the course of the pregnancy or pregnancy care; others will investigate adoption or choose to learn about the expected outcome, neonatal care, and long-term care for a child with disabilities.

- ACMG recommends:

- Informing all pregnant women that NIPS is the most sensitive screening option for traditionally screened aneuploidies (i.e., Patau, Edwards, and Down syndromes).

- Referring patients to a trained genetics professional when an increased risk of aneuploidy is reported after NIPS.

- Offering diagnostic testing when a positive screening test result is reported after NIPS.

- Providing accurate, balanced, up-to-date information, at an appropriate literacy level when a fetus is diagnosed with a chromosomal or genomic variation in an effort to educate prospective parents about the condition of concern. These materials should reflect the medical and psychosocial implications of the diagnosis ${ }^{41}$ (see Patient Resources).

- Laboratories should provide readily visible and clearly stated DR, SPEC, PPV, and NPV for conditions being screened, in pretest marketing materials, and when reporting laboratory results to assist patients and providers in making decisions and interpreting results.

- Laboratories should not offer screening for Patau, Edwards, and Down syndromes if they cannot report DR, SPEC, and PPV for these conditions.

\section{SHOULD NIPS BE USED TO SCREEN FOR AUTOSOMAL ANEUPLOIDIES OTHER THAN PATAU, EDWARDS, AND DOWN SYNDROMES?}

The expansion of NIPS to autosomes beyond 13, 18, and 21 is technically possible. Whole-chromosome fetal aneuploidy other than these common aneuploidies most often results in early fetal loss. ${ }^{42}$ Counseling related to these rare autosomal aneuploidies is made difficult by limited case reports and variable expressivity. Confined placental mosaicism for chromosome 16 has been well described and results in a spectrum of fetal outcomes from no clinical phenotype to fetal growth restriction. In a large retrospective study of amniocentesis performed for maternal age, ultrasound findings, biochemical abnormalities, or familial indications, $1 / 14,830$ patients had trisomy $2,8,12$, or $22 .{ }^{43}$ Detection of lethal chromosome abnormalities for which the natural course will be fetal loss has the potential to result in unnecessary diagnostic procedures and unnecessary pregnancy termination procedures. In addition to having a personal impact on patients, data collection in the public health sector could result in inflated pregnancy loss attributed to diagnostic procedures and maternal complications from pregnancy termination.

- ACMG does not recommend:

- NIPS to screen for autosomal aneuploidies other than those involving chromosomes 13, 18, and 21.

\section{HOW ARE NO-CALLS AVOIDED, INTERPRETED, AND MANAGED?}

\section{Fetal fraction}

The placental fraction accounts for approximately $10 \%$ of all cell-free DNA in maternal circulation. ${ }^{6,21,44}$ Data suggest that the lower limit of cell-free fetal DNA for a reliable result is approximately $4 \%$. A no-call may be reported if there is not a sufficient amount of fetal cell-free DNA in the maternal blood sample. In two prospective studies including more than 16,000 pregnancies, a low fetal fraction in maternal circulation was associated with an increased risk of fetal aneuploidies. ${ }^{24,45}$ The biologic mechanism of low fetal fraction and its association with aneuploidies is speculative. Interestingly, triploidy was most common (31\%); however, trisomy 21 was seen in $23 \%$ of cases of low fetal fraction. ${ }^{24}$ Others showed that a fetal fraction of DNA in Down syndrome cases is often the same or higher when compared to pregnancies with euploid fetuses. ${ }^{46,47}$ Since the introduction of NIPS into clinical practice, fetal fraction has not been uniformly reported by laboratories. The described relationship between low fetal fraction and increased risk of aneuploidy adds to the importance of reporting the reason for a no-call and of indicating in the report whether a low fetal fraction was identified.

Factors that influence fetal fraction include maternal weight and gestational age. ${ }^{47-49}$ There is no specific threshold to describe the relationship between fetal fraction and maternal weight. However, in cases of significant obesity, a no-call due to low fetal fraction should be anticipated. There is a gestational age threshold, below which results are not reliable (9 or 10 weeks depending on the laboratory used). Data suggest that before 20 weeks, fetal fraction increases less than $0.1 \%$ per week, which challenges the idea that repeating sample collection is a viable approach to overcoming a low fetal fraction. ${ }^{47,49}$ 
- ACMG recommends:

- Offering diagnostic testing for a no-call NIPS result due to low fetal fraction if maternal blood for NIPS was drawn at an appropriate gestational age. A repeat blood draw is NOT appropriate.

$\circ$ Offering aneuploidy screening other than NIPS in cases of significant obesity.

○ All laboratories should include a clearly visible fetal fraction on NIPS reports.

○ All laboratories should establish and monitor analytical and clinical validity for fetal fraction.

- All laboratories should specify the reason for a no-call when reporting NIPS results.

\section{Long stretches of homozygosity}

Single-nucleotide polymorphisms or array-based assays require adequate heterozygosity between the maternal and fetal genomes to provide meaningful data for the analysis of genomic balance and copy number. Therefore, stretches of homozygosity between the maternal and fetal genomes render any differences in copy number within that region undetectable, including small duplications or deletions. In addition to preventing in the interpretation of genomic balance, large regions of homozygosity for a single chromosome may be suggestive of uniparental disomy (UPD), whereas large regions of homozygosity dispersed over many chromosomes may be suggestive of parental consanguinity. ${ }^{50}$

- ACMG recommends:

- Informing patients that a no-call result may be due to long stretches of homozygosity, which could be due to either UPD or parental consanguinity.

- Referring patients to a trained genetics professional when a no-call result suspicious for UPD or parental consanguinity is received.

- Offering diagnostic testing with CMA when a no-call result is obtained after NIPS due to possible UPD or parental consanguinity.

\section{SHOULD NIPS BE OFFERED TO SCREEN FOR SEX CHROMOSOME ANEUPLOIDIES?}

In one retrospective study of 88,970 amniocenteses, the diagnosis of any sex chromosome aneuploidy was made in $1 / 272$ patients. ${ }^{43}$ This was higher for women older than 35 years compared to younger women (1/210 and $1 / 459$, respectively). Conventional screening for aneuploidies does not detect sex chromosome aneuploidies. The most common of these, monosomy X (Turner syndrome), has been estimated to occur in $1-1.5 \%$ of pregnancies ${ }^{51}$ and is a common cause of firsttrimester pregnancy loss $(\sim 23 \%){ }^{52}$ The phenotype of individuals with a $47, \mathrm{XXX}$ or $47, \mathrm{XYY}$ karyotype is highly variable but may include social or cognitive deficits. ${ }^{53}$ Klinefelter syndrome $(47, \mathrm{XXY})$, however, does have a classic phenotype and is associated with sterility. ${ }^{53}$

The detection rate (clinical validity) of sex chromosome aneuploidies after NIPS is reported to be more than $90 \%$ and has a false-positive rate of approximately $1 \%{ }^{54-57}$ The PPV (clinical utility) for the aggregate of sex chromosome aneuploidies among prospectively collected samples was $48.4 \%$ (range for specific aneuploidies, 30-67\%). ${ }^{57} \mathrm{~A}$ PPV in these ranges is considerably higher than those accepted for conventional screening of Patau, Edwards, and Down syndromes.

Etiologies of false-positive sex chromosome aneuploidy results have been considered, and an approach to distinguish true positives from false positives was described. ${ }^{58}$ Maternal medical, endocrine, and fertility history can help to identify the cause of a false-positive result. This includes patients with an organ transplantation from either a 46,XY individual or unknown gender donor. Other causes of false-positive results are similar to those for traditional aneuploidies. These include confined placental mosaicism, "vanishing" twin or higher-order co-fetus, and, rarely, maternal neoplasm. For these reasons, patients should be counseled about the advantages and disadvantages of sex chromosome aneuploidy screening within the construct of their preferences for information.

- ACMG recommends:

○ Informing all pregnant women, as part of pretest counseling for NIPS, of the availability of the expanded use of screening for sex chromosome aneuploidies.

- Providers should make efforts to deter patients from selecting sex chromosome aneuploidy screening for the sole purpose of biologic sex identification in the absence of a clinical indication for this information.

- Informing patients about the causes and increased possibilities of false-positive results for sex chromosome aneuploidies as part of pretest counseling and screening for these conditions. Patients should also be informed of the potential for results of conditions that, once confirmed, may have a variable prognosis (e.g., Turner syndrome) before consenting to screening for sex chromosome aneuploidies.

- Referring patients to a trained genetics professional when an increased risk of sex chromosome aneuploidy is reported after NIPS.

- Offering diagnostic testing when a positive screening test result is reported after screening for sex chromosome aneuploidies.

- Providing accurate, balanced, up-to-date information and materials at an appropriate literacy level when a fetus is diagnosed with a sex chromosome aneuploidy in an effort to educate prospective parents about the specific condition. These materials should reflect medical and psychosocial implications for the diagnosis ${ }^{41}$ (see Patient Resources).

- Laboratories include easily recognizable and highly visible DR, SPEC, PPV, and NPV for each sex chromosome aneuploidy when reporting results to assist patients and providers in making decisions and interpreting results.

- Laboratories should not offer screening for sex chromosome aneuploidies if they cannot report DR, SPEC, PPV, and NPV for these conditions. 


\section{SHOULD NIPS BE OFFERED FOR DETECTION OF COPY NUMBER VARIATION (CNV)?}

Conventional aneuploidy screening focuses on wholechromosome aneuploidies that have an overall live birth frequency of $1 / 800$ (Down syndrome) ${ }^{59}$ to $1 / 30,000$ (Patau syndrome). Expanding NIPS to include detection of specific conditions caused by a CNV (e.g., 22q11.2 deletion, 1p36 deletion, 15q11.2-13 deletion) is technically possible (analytical validity) ${ }^{60-63}$ The phenotypes associated with these conditions can be severe; therefore, they may be appropriate conditions for prenatal screening. However, providers and patients must be aware that expanding the use of NIPS to include the detection of CNVs requires in-depth knowledge of the limitations of the technology, return of results, and follow-up.

Validation studies indicate a high detection rate $(>97 \%)$ and low false-positive rate $(<1 \%)$ can be achieved. However, there are few clinical utility studies. Therefore, PPV and NPV have been modeled. ${ }^{63-65}$ One report showed that for a specific combination of CNVs studied, PPV ranged from 3.8 to $17 \%$. In a large retrospective study of more than 21,000 samples, the aggregate PPV for several CNVs screened simultaneously was 18\% (specific conditions: $11-48 \%$ ). Methods to improve PPV have been reported. ${ }^{65}$ Modeling PPV and NPV is made more complex for genome-wide analysis for which validation studies are limited in scope and number. ${ }^{26,63}$ Determination of PPV and NPV is hampered by the inherent limitations of studying multiple rare conditions with variable expressivity. As greater portions of the genome are analyzed for CNVs, false positive and negative results are expected to increase. This may result in an increase in patient anxiety and fetal procedures and a burden on an already limited genetic counseling workforce.

Validation studies make the point that DR and SPEC depend on many variables (e.g., depth of read), ${ }^{10,60-63}$ which can change the false-positive and false-negative rate when NIPS is used for prenatal detection of CNVs. Pretest and posttest counseling is further confounded by variable expressivity and penetrance of the conditions being screened, size of the deletion being screened, specific genes within the critical region of the locus interrogated, and the number of genes within the critical region being screened.

- ACMG recommends:

O Informing all pregnant women of the availability of the expanded use of NIPS to screen for clinically relevant CNVs when the following conditions can also be met:

- Obstetric care providers should discuss with their patients the desire for prenatal screening as opposed to diagnostic testing (i.e., CVS or amniocentesis).

- Obstetric care providers should discuss with their patients the desire for maximum fetal genomic information through prenatal screening.

- Obstetric care providers should inform their patients of the higher likelihood of false-positive and false-negative results for these conditions as compared to results obtained when NIPS is limited to common aneuploidy screening.

- Obstetric care providers should inform their patients of the potential for results of conditions that, once confirmed, may have an uncertain prognosis.

- Referring patients to a trained genetics professional when NIPS identifies a CNV.

- Offering diagnostic testing (CVS or amniocentesis) with CMA when NIPS identifies a CNV.

- Providing accurate, balanced, up-to-date information at an appropriate literacy level when a fetus is diagnosed with a CNV in an effort to educate prospective parents about the condition of concern. These materials should reflect the medical and psychosocial implications of the diagnosis ${ }^{65}$ (see Patient Resources).

- Laboratory requisitions and pretest counseling information should specify the DR, SPEC, PPV, and NPV of each CNV screened. This material should state whether PPV and NPV are modeled or derived from clinical utility studies (natural population or sample with known prevalence).

- Laboratories include easily recognizable and highly visible DR, SPEC, PPV, and NPV for each CNV screened when reporting laboratory results to assist patients and providers in making decisions and interpreting results. Reports should state whether PPV and NPV are modeled or derived from clinical utility studies (natural population or sample with known prevalence). When laboratories cannot report specific DR, SPEC, PPV, and NPV, screening for those CNVs should not be performed by that laboratory.

- $\quad$ ACMG does not recommend:

○ NIPS to screen for genome-wide CNVs. If this level of information is desired, then diagnostic testing (e.g., chorionic villous sampling or amniocentesis) followed by CMA is recommended.

\section{SPECIAL CONSIDERATIONS}

\section{Multiple gestation and/or donor oocytes:}

There are unique challenges when NIPS is used in multiple gestation pregnancies conceived through donor oocytes. These are specific to the analytical method and bioinformatics employed by the laboratory.

- ACMG recommends:

O In pregnancies with multiple gestations and/or donor oocytes, testing laboratories should be contacted regarding the validity of NIPS before it is offered to the patient as a screening option.

\section{Unanticipated findings}

Both constitutional and acquired forms of genomic imbalance in the mother (e.g., aneuploidy of chromosome X, microdeletions, neoplasia, chimerism due to allogenic organ 
or tissue transplantation, or mosaicism) and imbalances within the fetoplacental genome (e.g., confined placental mosaicism) can give rise to identifiable bioinformatic patterns that may confound interpretations. Therefore, providers should be aware of the potential for false-positive results that may resolve after diagnostic testing. Although it is not the purpose of NIPS to identify clinically relevant maternal genomic information, patients and providers should be aware of the potential for inadvertent discovery of such information and the potential for additional follow-up testing unrelated to the pregnancy.

Given the differences in laboratory methodologies and bioinformatic processing that may be used, it is beyond the scope of this document to address considerations that might be unique to any specific method in use. It therefore remains the responsibility of each laboratory to make physician providers aware of clinically relevant features that are specific to the methodology used. This is best accomplished through educational materials and laboratory reports.

- ACMG recommends:

O Informing patients of the possibility of identifying maternal genomic imbalances and that this possibility depends on the specific methodology used.

o Referring patients to a trained genetics professional when NIPS identifies maternal genomic imbalances.

O Offering aneuploidy screening other than NIPS for patients with a history of bone marrow or organ transplantation from a male donor or donor of uncertain biologic sex.

- Discussing the possibility of discordant fetal biologic sex if maternal blood transfusion was performed $<4$ weeks prior to the blood draw for NIPS.

\section{Positive and negative predictive values}

Understanding the importance of PPV is paramount to screening. PPV is a screening test metric that is useful when patients screen positive. This metric is used by patients in deciding the next steps in decision making. Because the specificity is so high after NIPS for traditionally screened aneuploidies, NPV is less often the focus. However, it is one of the key features of this technology. A high NPV offers patients reassurance in the posttest setting. There are several mathematical approaches that can be used to model PPV and NPV from validation data. PPV for aneuploidy is very sensitive to prevalence/a priori risk, and to a lesser extent DR and SPEC, which do not fluctuate with maternal age. Maternal age is a highly important factor in determining the prevalence of Down syndrome and other aneuploidies, but it is not a factor when considering CNVs. One reason why $\mathrm{PPV}$ is much lower for detection of CNVs is that the prevalence and detection rate are low compared to traditionally screened aneuploidies. A common error is to interpret PPV across an entire population without taking into account patient-specific information (e.g., prevalence based on maternal age when necessary).
There are several online calculators for determining patient-specific PPV and NPV after NIPS (e.g., http://secure. itswebs.com/nsgc/niptcalculator/index.html). PPV seems irrelevant to anyone not facing a positive test result. If the PPV of each condition being considered were reported when results were negative, then there would be an excess of data cluttering a report.

- ACMG recommends:

O Laboratories provide patient-specific PPV when reporting positive test results.

- Laboratories provide population-derived PPV when reporting positive results in cases in which patientspecific PPV cannot be determined due to unavailable clinical information.

- Laboratories provide modeled PPV when reporting positive results for which neither patient-specific nor population-derived PPV are possible.

o Providers use validated online calculators to provide patient-specific PPV when results from NIPS are positive to facilitate clear and accurate communication with patients.

O Incorporating laboratory-specific DR and SPEC to provide clear and patient-specific information when using validated online calculators.

\section{PATIENT RESOURCES}

In a consensus statement by the ACMG, the American College of Obstetricians and Gynecologists (ACOG), the National Society of Genetics Counselors (NSGC), and Down syndrome organizations, there was unanimous agreement that patient education materials about prenatal testing and associated conditions should result from "collaboration among healthcare and advocacy organizations." 41 According to Public Law 110-371 (https://www.govtrack.us/congress/bills/110/s1810/text), enacted in 2008, "partnerships between healthcare professional groups and disability advocacy organizations" were emphasized regarding the collection, synthesis, and dissemination of "current evidence-based information" related to prenatal conditions. With these charges in mind, the ACMG has identified available patient resources (listed alphabetically) that have resulted from collaborations between healthcare professional groups and advocacy organizations.

Down Syndrome Pregnancy (http://downsyndromepregnancy.org/books). This site, for expectant parents preparing for the birth of a baby with Down syndrome, provides a range of books in English and Spanish that are recommended in the "NSGC Guidelines for Communicating a Prenatal or Postnatal Diagnosis of Down Syndrome" and that have been reviewed by medical and patient advocacy experts.

Genetics Home Reference (https://ghr.nlm.nih.gov). This online reference provides information for patients and families about more than 1,000 genetic conditions. All content is written by a full-time staff with backgrounds in genetics, reviewed by outside experts, and contains input from support and advocacy 
organizations. Genetics Home Reference is a service of the National Library of Medicine, which is part of the National Institutes of Health, an agency of the US Department of Health and Human Services.

Genetic Support Foundation (https://www.geneticsupportfoundation.org). This nonprofit organization, founded by genetics professionals, provides information about pregnancy and genetics and the different conditions that can be detected prenatally. It often includes instructional videos.

Lettercase/The National Center for Prenatal and Postnatal Resources (http://www.lettercase.org). Lettercase offers professionally reviewed materials about genetic conditions. Currently, "Understanding a Down Syndrome Diagnosis" and "Understanding a Turner Syndrome Diagnosis" are available in print and digital versions in several languages. The materials are intended for expectant couples who have received a prenatal diagnosis of Down or Turner syndrome but have not yet made a decision regarding their pregnancy options. The materials are prepared with assistance from the ACMG, ACOG, NSGC, and national patient advocacy organizations.

NSGC "Fact Sheet about Down Syndrome for New and Expectant Parents" (http://nsgc.org/p/cm/ld/fid=387) and “A Patient's Guide to Understanding Noninvasive Prenatal Testing" (http://nsgc.org/p/cm/ld/fid=385). These fact sheets on the NSGC website, which provide basic downloadable information, were reviewed by the National Society of Genetic Counselors Down Syndrome Information Act Working Group, with assistance from the National Center for Prenatal and Postnatal Resources.

\section{PROVIDER RESOURCES}

The following resources (listed alphabetically) were created by respected medical organizations or medical expert consensus and can serve as useful references for medical providers.

Delivering a diagnosis. Resources describing simulation training for healthcare professionals who deliver a prenatal diagnosis to expectant couples are available. These projects were funded by federal grants and efficacy was researched and published. ${ }^{66,67}$

Down syndrome healthcare guidelines. "Healthcare Supervision for Children with Down Syndrome" (http:// pediatrics.aappublications.org/content/128/2/393). This was written by the Committee on Genetics of the American Academy of Pediatrics, provides guidance for healthcare professionals. Resources for parents are also listed.

GeneReviews (http://www.ncbi.nlm.nih.gov/books/ NBK1116). This online resource for clinicians provides peerreviewed information written by medical experts. Information is updated every 2 to 4 years through a formal review process. It is an excellent source of information, and physicians faced with a need to learn about common CNVs may find this resource useful.
"Care of Girls and Women with Turner Syndrome: A Guideline of the Turner Syndrome Study Group." This was written by the Turner Syndrome Consensus Study Group of the National Institutes of Health and was adopted by the American Academy of Pediatrics. ${ }^{68}$

22q11 deletion syndrome (DiGeorge syndrome) guidelines. Peer-reviewed expert consensus documents are available for the evaluation and management of patients with 22q11 deletion syndrome (DiGeorge syndrome) ${ }^{69,70}$ This is the most common copy-number variation currently being offered through NIPS. Resources for other CNVs may be found in GeneReviews.

\section{SUMMARY}

New data and provider and patient demands require an updated position on the use of NIPS in prenatal care. We provide a framework for understanding how genetic technology moves from an idea into clinical practice. We hope this framework helps to explain ACMG's recommendations. Clinical validation strongly suggested that NIPS can replace conventional screening for Patau, Edwards, and Down syndromes. Objective measures of clinical utility support this. Test metrics support NIPS across the maternal age spectrum and continuum of gestational age beginning at 9-10 weeks as long as patients are not significantly obese. In the latter case, fetal fraction leading to an inability to make a call is limiting.

We have raised the bar for pretest counseling by expanding NIPS beyond that for Patau, Edwards, and Down syndromes. Providers should have a thorough understanding of patient preferences; efforts to educate about the limitations are not trivial. Although clinical utility studies are limited, they point to a role for NIPS in sex chromosome aneuploidy screening and screening for selected CNVs. We support these uses when the live birth frequency of conditions reaches or exceeds that of currently screened conditions and when test metrics meet or exceed those of wellestablished approaches to prenatal screening. Furthermore, we considered the potential for children to be impacted by early treatment. Our recommendations will affect communication between providers and patients and between providers and testing laboratories. Laboratories are encouraged to meet the needs of providers and patients by delivering meaningful screening reports, engaging in education, and identifying ways to address distributive justice, a medical ethical principle that challenges genomics-based innovative and clinically useful technologies.

\section{ACKNOWLEDGMENT}

The ACMG Noninvasive Prenatal Screening Work Group is grateful to Marsha Harben of the University of Florida for her tireless assistance in the preparation of this document.

\section{DISCLOSURE}

B.G.S. serves on the Advisory Board of several nonprofit entities providing education about Down syndrome. The other authors declare no conflict of interest. 


\section{REFERENCES}

1. Driscoll DA, Gross SJ; Professional Practice and Guidelines Committee. First trimester diagnosis and screening for fetal aneuploidy. Genet Med 2008;10: 73-75.

2. Grody WW, Thompson BH, Gregg AR, et al. ACMG position statement on prenatal/ preconception expanded carrier screening. Genet Med 2013;15:482-483.

3. Gregg AR, Gross SJ, Best RG, et al. ACMG statement on noninvasive prenatal screening for fetal aneuploidy. Genet Med 2013;15:395-398.

4. Palomaki GE, Kloza EM, Lambert-Messerlian GM, et al. DNA sequencing of maternal plasma to detect Down syndrome: an international clinical validation study. Genet Med 2011;13:913-920.

5. Ehrich M, Deciu C, Zwiefelhofer T, et al. Noninvasive detection of fetal trisomy 21 by sequencing of DNA in maternal blood: a study in a clinical setting. Am J Obstet Gynecol 2011;204:205.e1-205.11.

6. Norton ME, Brar H, Weiss J, et al. Non-Invasive Chromosomal Evaluation (NICE) Study: results of a multicenter prospective cohort study for detection of fetal trisomy 21 and trisomy 18. Am J Obstet Gynecol 2012;207:137.e1137.e8.

7. Bianchi DW, Platt LD, Goldberg JD, Abuhamad AZ, Sehnert AJ, Rava RP. MatErnal BLood IS Source to Accurately diagnose fetal aneuploidy (MELISSA) Study Group. Genome-wide fetal aneuploidy detection by maternal plasma DNA sequencing. Obstet Gynecol 2012;119:890-901.

8. Nicolaides KH, Syngelaki A, Gil M, Atanasova V, Markova D. Validation of targeted sequencing of single-nucleotide polymorphisms for non-invasive prenatal detection of aneuploidy of chromosomes 13, 18, 21, X, and Y. Prenat Diagn 2013;33:575-579.

9. Liao $C$, Yin $A H$, Peng $C F$, et al. Noninvasive prenatal diagnosis of common aneuploidies by semiconductor sequencing. Proc Natl Acad Sci USA 2014;111:7415-7420.

10. Stokowski R, Wang E, White $K$, et al. Clinical performance of non-invasive prenatal testing (NIPT) using targeted cell-free DNA analysis in maternal plasma with microarrays or next generation sequencing (NGS) is consistent across multiple controlled clinical studies. Prenat Diagn 2015;35:1243-1246.

11. Skirton H, Goldsmith L, Jackson L, Lewis C, Chitty LS. Non-invasive prenatal testing for aneuploidy: a systematic review of Internet advertising to potential users by commercial companies and private health providers. Prenat Diagn 2015;35:1167-1175.

12. Lutgendorf MA, Stoll KA, Knutzen DM, Foglia LM. Noninvasive prenatal testing: limitations and unanswered questions. Genet Med 2014;16:281-285.

13. Benn P, Borrell A, Chiu RW, et al. Position statement from the Chromosome Abnormality Screening Committee on behalf of the Board of the International Society for Prenatal Diagnosis. Prenat Diagn 2015;35:725-734.

14. Wilson KL, Czerwinski JL, Hoskovec JM, et al. NSGC practice guideline: prenatal screening and diagnostic testing options for chromosome aneuploidy. J Genet Couns 2013;22:4-15.

15. ACOG practice bulletin number 163: screening for fetal aneuploidy. Obstet Gynecol 2016;127:e123-137.

16. Fan HC, Quake SR. Detection of aneuploidy with digital polymerase chain reaction. Anal Chem 2007;79:7576-7579.

17. Lo YM, Corbetta N, Chamberlain PF, et al. Presence of fetal DNA in maternal plasma and serum. Lancet 1997;350:485-487.

18. Lo YM, Lun FM, Chan KC, et al. Digital PCR for the molecular detection of fetal chromosomal aneuploidy. Proc Natl Acad Sci USA 2007;104:13116-13121.

19. Fan HC, Blumenfeld YJ, Chitkara U, Hudgins L, Quake SR. Noninvasive diagnosis of fetal aneuploidy by shotgun sequencing DNA from maternal blood. Proc Natl Acad Sci USA 2008;105:16266-16271.

20. Ashoor G, Syngelaki A, Wagner M, Birdir C, Nicolaides KH. Chromosomeselective sequencing of maternal plasma cell-free DNA for first-trimester detection of trisomy 21 and trisomy 18. Am J Obstet Gynecol 2012;206:322. e1-322.e5.

21. Sparks AB, Struble CA, Wang ET, Song K, Oliphant A. Noninvasive prenatal detection and selective analysis of cell-free DNA obtained from maternal blood: evaluation for trisomy 21 and trisomy 18. Am J Obstet Gynecol 2012;206:319. e1-319.e9.

22. Zimmermann B, Hill M, Gemelos $G$, et al. Noninvasive prenatal aneuploidy testing of chromosomes $13,18,21, \mathrm{X}$, and $\mathrm{Y}$, using targeted sequencing of polymorphic loci. Prenat Diagn 2012;32:1233-1241.

23. Bianchi DW, Parker RL, Wentworth J, et al.; CARE Study Group. DNA sequencing versus standard prenatal aneuploidy screening. N Engl J Med 2014;370:799-808.

24. Norton ME, Jacobsson B, Swamy GK, et al. Cell-free DNA analysis for noninvasive examination of trisomy. N Engl J Med 2015;372: 1589-1597.
25. Zhang $H$, Gao $Y$, Jiang $F$, et al. Non-invasive prenatal testing for trisomies 21, 18 and 13: clinical experience from 146,958 pregnancies. Ultrasound Obstet Gynecol 2015;45:530-538.

26. Kaimal AJ, Norton ME, Kuppermann M. Prenatal Testing in the Genomic Age: Clinical Outcomes, Quality of Life, and Costs. Obstet Gynecol 2015;126: 737-746.

27. Edwards JG, Feldman G, Goldberg J, et al. Expanded carrier screening in reproductive medicine-points to consider: a joint statement of the American College of Medical Genetics and Genomics, American College of Obstetricians and Gynecologists, National Society of Genetic Counselors, Perinatal Quality Foundation, and Society for Maternal-Fetal Medicine. Obstet Gynecol 2015;125:653-662.

28. Benkendorf JL, Callanan NP, Grobstein R, Schmerler S, FitzGerald KT. An explication of the National Society of Genetic Counselors (NSGC) code of ethics. J Genet Couns 1992;1:31-39.

29. Bennett RL, Callanan N, Gordon E, et al.; The National Society of Genetic Counselors Code of Ethics Work Group. Code of ethics of the National Society of Genetic Counselors: explication of revisions. J Genet Couns 2006;15: 313-323.

30. Rogers CR. Counseling and Psychotherapy. Houghton Mifflin: Cambridge, MA, 1942.

31. Elias S, Annas GJ. Generic consent for genetic screening. N Engl J Med 1994;330:1611-1613.

32. Driscoll DA, Gross SJ; Professional Practice Guidelines Committee. Screening for fetal aneuploidy and neural tube defects. Genet Med 2009;11:818-821.

33. Guttmacher Institute. An overview of abortion laws. http://www.guttmacher. org/state-policy/explore/overview-abortion-laws. Accessed 4 January 2016.

34. Wapner RJ, Martin CL, Levy B, et al. Chromosomal microarray versus karyotyping for prenatal diagnosis. N Engl J Med 2012;367:2175-2184.

35. Akolekar R, Beta J, Picciarelli G, Ogilvie C, D'Antonio F. Procedure-related risk of miscarriage following amniocentesis and chorionic villus sampling: a systematic review and meta-analysis. Ultrasound Obstet Gynecol 2015;45: 16-26.

36. Mujezinovic F, Alfirevic Z. Procedure-related complications of amniocentesis and chorionic villous sampling: a systematic review. Obstet Gynecol 2007;110: 687-694.

37. Guedj F, Bianchi DW, Delabar JM. Prenatal treatment of Down syndrome: a reality? Curr Opin Obstet Gyneco/ 2014;26:92-103.

38. Committee Opinion No. 640: cell-free DNA screening for fetal aneuploidy. Obstet Gynecol 2015, 126:e31-37.

39. SMFM Statement: clarification of recommendations regarding cell-free DNA aneuploidy screening. Am J Obstet Gyneco/ 2015;213:753-754.

40. Benn P, Borell A, Chiu R, et al. Position statement from the Aneuploidy Screening Committee on behalf of the Board of the International Society for Prenatal Diagnosis. Prenat Diagn 2013;33:622-629.

41. Edwards JG, Ferrante RR. Understanding prenatal screening and diagnosis of Down syndrome from the health professional and advocacy community perspectives. 2009. https://www.acmg.net/StaticContent/Resources/ Consensus\%20Conversation\%20Statement.pdf.

42. Nussbaum RL, McInnes RR, Willard HF. Thompson \& Thompson Genetics in Medicine. 8th edition. Elsevier: Philadelphia, PA, 2007.

43. Forabosco A, Percesepe A, Santucci S. Incidence of non-age-dependent chromosomal abnormalities: a population-based study on 88965 amniocenteses. Eur J Hum Genet 2009;17:897-903.

44. Ashoor G, Syngelaki A, Poon LC, Rezende JC, Nicolaides KH. Fetal fraction in maternal plasma cell-free DNA at 11-13 weeks' gestation: relation to maternal and fetal characteristics. Ultrasound Obstet Gynecol 2013;41:26-32.

45. Pergament E, Cuckle H, Zimmermann B, et al. Single-nucleotide polymorphismbased noninvasive prenatal screening in a high-risk and low-risk cohort. Obstet Gynecol 2014;124(2 Pt 1):210-218.

46. Rava RP, Srinivasan A, Sehnert AJ, Bianchi DW. Circulating fetal cell-free DNA fractions differ in autosomal aneuploidies and monosomy X. Clin Chem 2014;60:243-250.

47. Kinnings SL, Geis JA, Almasri E, et al. Factors affecting levels of circulating cell-free fetal DNA in maternal plasma and their implications for noninvasive prenatal testing. Prenat Diagn 2015;35:816-822.

48. Wataganara T, Peter I, Messerlian GM, Borgatta L, Bianchi DW. Inverse correlation between maternal weight and second trimester circulating cell-free fetal DNA levels. Obstet Gyneco/ 2004:104:545-550.

49. Wang E, Batey A, Struble C, Musci T, Song K, Oliphant A. Gestational age and maternal weight effects on fetal cell-free DNA in maternal plasma. Prenat Diagn 2013;33:662-666. 
50. Rehder CW, David KL, Hirsch B, Toriello HV, Wilson CM, Kearney HM. American College of Medical Genetics and Genomics: standards and guidelines for documenting suspected consanguinity as an incidental finding of genomic testing. Genet Med 2013;15:150-152.

51. Hook EB, Warburton $D$. Turner syndrome revisited: review of new data supports the hypothesis that all viable $45, X$ cases are cryptic mosaics with a rescue cell line, implying an origin by mitotic loss. Hum Genet 2014;133:417-424.

52. Pauli RM, Reiser CA, Lebovitz RM, Kirkpatrick SJ. Wisconsin Stillbirth Service Program: I. Establishment and assessment of a community-based program for etiologic investigation of intrauterine deaths. Am J Med Genet 1994;50: 116-134.

53. Jones KL, Jones MC, del Campo M. Smith's Recognizable Patterns of Human Malformation. Paalman MH, Carey JC, eds. Elsevier Saunders: Philadelphia, PA, 2013. 7:7-83.

54. Mazloom AR, Džakula Ž, Oeth $P$, et al. Noninvasive prenatal detection of sex chromosomal aneuploidies by sequencing circulating cell-free DNA from maternal plasma. Prenat Diagn 2013;33:591-597.

55. Samango-Sprouse C, Banjevic M, Ryan A, et al. SNP-based non-invasive prenatal testing detects sex chromosome aneuploidies with high accuracy. Prenat Diagn 2013:33:643-649.

56. Nicolaides KH, Musci TJ, Struble CA, Syngelaki A, Gil MM. Assessment of fetal sex chromosome aneuploidy using directed cell-free DNA analysis. Fetal Diagn Ther 2014;35:1-6.

57. Porreco RP, Garite TJ, Maurel K, et al.; Obstetrix Collaborative Research Network. Noninvasive prenatal screening for fetal trisomies 21, 18, 13 and the common sex chromosome aneuploidies from maternal blood using massively parallel genomic sequencing of DNA. Am J Obstet Gynecol 2014;211:365.e1-365.12.

58. Bianchi DW, Parsa S, Bhatt $S$, et al. Fetal sex chromosome testing by maternal plasma DNA sequencing: clinical laboratory experience and biology. Obstet Gynecol 2015;125:375-382.

59. de Graaf G, Buckley F, Skotko BG. Live births, natural losses, and elective terminations with Down syndrome in Massachusetts. Genet Med 2016;18:459-466.
60. Peters D, Chu T, Yatsenko SA, et al. Noninvasive prenatal diagnosis of a fetal microdeletion syndrome. N Engl J Med 2011;365:1847-1848.

61. Srinivasan A, Bianchi DW, Huang H, Sehnert AJ, Rava RP. Noninvasive detection of fetal subchromosome abnormalities via deep sequencing of maternal plasma. Am J Hum Genet 2013;92:167-176.

62. Zhao C, Tynan J, Ehrich M, et al. Detection of fetal subchromosomal abnormalities by sequencing circulating cell-free DNA from maternal plasma. Clin Chem 2015:61:608-616.

63. Lefkowitz RB, Tynan JA, Liu T, et al. Clinical validation of a noninvasive prenatal test for genomewide detection of fetal copy number variants. Am J Obstet Gyneco/ 2016; e-pub ahead of print 17 February 2016.

64. Wapner RJ, Babiarz JE, Levy B, et al. Expanding the scope of noninvasive prenatal testing: detection of fetal microdeletion syndromes. Am J Obstet Gynecol 2015;212:332.e1-332.e9.

65. Gross SJ, Ryan A, Benn P. Noninvasive prenatal testing for $22 q 11.2$ deletion syndrome: deeper sequencing increases the positive predictive value. Am J Obstet Gynecol 2015;213:254-255

66. Ferguson JE 2nd, Kleinert HL, Lunney CA, Campbell LR. Resident physicians' competencies and attitudes in delivering a postnatal diagnosis of Down syndrome. Obstet Gynecol 2006;108:898-905.

67. Lunney CA, Kleinert HL, Ferguson JE 2nd, Campbell L. Effectively training pediatric residents to deliver diagnoses of Down syndrome. Am J Med Genet $A$ 2012;158A:384-390.

68. Bondy CA; Turner Syndrome Study Group. Care of girls and women with Turner syndrome: a guideline of the Turner Syndrome Study Group. J Clin Endocrinol Metab 2007:92:10-25.

69. Bassett AS, McDonald-McGinn DM, Devriendt K, et al.; International 22q11.2 Deletion Syndrome Consortium. Practical guidelines for managing patients with 22q11.2 deletion syndrome. J Pediatr 2011;159:332-9.e1.

70. Habel A, Herriot R, Kumararatne D, et al. Towards a safety net for management of 22q11.2 deletion syndrome: guidelines for our times. Eur J Pediatr 2014:173:757-765 